\title{
Effects of ambient humidity on the Cochet-Bonnet aesthesiometer
}

\author{
Edward Lum ${ }^{1,2}$ - Paul J. Murphy ${ }^{1,3}$
}

Received: 5 September 2017 / Revised: 11 January 2018 / Accepted: 16 May 2018 / Published online: 19 June 2018

(c) The Royal College of Ophthalmologists 2018

\begin{abstract}
Purpose The Cochet-Bonnet (COBO) aesthesiometer is the current standard in corneal sensitivity assessment. This study investigates the influence of ambient room humidity levels on the stimulus force exerted by the instrument.

Methods A COBO instrument (Luneau Opthalmologie) with $0.12 \mathrm{~mm}$ nominal nylon filament diameter was placed in an environment chamber (Electro-tech systems Inc. PA, USA) at $25^{\circ} \mathrm{C}$ and relative humidity (\%RH) set to either $20-80 \%$, in $10 \%$ steps. After $12 \mathrm{~h}$ in the chamber at a chosen $\% \mathrm{RH}$ level, the instrument was removed and exerted force measured by pressing the nylon filament onto the plate of an analytical microbalance (Mettler-Toledo AB265; precision $\pm 0.0001 \mathrm{~g}$ ) at a perpendicular angle, by a predetermined amount. Exerted force onto the microbalance was recorded in grams for a specified filament length. Procedure was repeated for filament lengths $10-60 \mathrm{~mm}$, in $5 \mathrm{~mm}$ steps. The instrument was returned to the chamber and procedure repeated 5 times, before repeating at the next \% RH setting (random order). Measurements at each filament lengths were compared using one-way ANOVA and post-hoc Tukey's range test. A $p$-value $<0.05$ denoted statistical significance.

Results Significant differences in exerted force were observed with alteration in \%RH levels for each filament length (all $p<$ 0.001 ). Exerted force decreased significantly with an increase in $\% \mathrm{RH}$ for all filament lengths, with the average force decreasing by $15 \%$ with each $10 \%$ rise in $\% \mathrm{RH}$.

Conclusions This study confirms previous suggestions that the rigidity of the COBO nylon filament is affected by ambient room humidity levels, with implications on the stimulus force delivered by the instrument. A conversion table is provided for converting filament lengths to pressure for a range of relative humidity levels.
\end{abstract}

\section{Introduction}

The primary role of the corneal innervation is to detect foreign bodies and noxious substances that come in contact with the eye. The dense neural network at the corneal surface provides a high level of sensitivity that also plays a primary role in the regulation of basal tears via the lacrimal function unit $[1,2]$. The assessment of corneal sensitivity can provide an indication of neural functioning, which,

Edward Lum

e.lum@unsw.edu.au

1 University of Waterloo, School of Optometry and Vision Science, Waterloo, ON, Canada

2 University of New South Wales, School of Optometry and Vision Science, Sydney, Australia

3 Cardiff University, School of Optometry and Vision Science, Cardiff, UK when compromised, can lead to disruptions in the trophic maintenance and repair of the corneal epithelium [3, 4].

Corneal sensitivity in humans is assessed using a contact method, as in the Cochet-Bonnet (COBO) aesthesiometer, or by non-contact methods, as with the Belmonte [5] aesthesiometer and Non-Contact Corneal Aesthesiometer [6]. Stimulation of nerve endings immediately beneath the corneal surface is achieved by directing either a nylon filament tip or a controlled gas-jet onto the corneal surface, during $\mathrm{COBO}$ and non-contact aesthesiometry, respectively. Although the range of force exerted by $\mathrm{COBO}$ is extremely low $(0.02-6 \mathrm{mN})$ [7], contact with the cornea by the filament tip commonly causes injury to the corneal epithelium during threshold measurements [8]. Despite this invasive design and other instrument limitations [9], the COBO continues to be considered the standard for corneal sensitivity assessment, as demonstrated in recent investigations involving ocular diseases [10-12], ocular surgery [13-16], and contact lens wear [17, 18], arguably because of the instrument's ease of use and commercial availability. 
Measurement of corneal sensitivity threshold can be performed using the COBO with either a $0.08 \mathrm{~mm}$ or 0.12 $\mathrm{mm}$ nominal diameter nylon filament. Although the thinner diameter filament offers a greater range of low stimulus intensities, its use in studies compared to the thicker filament is less frequent, presumably due to greater filament bending and movement when held in position during corneal sensitivity assessments, and lack of commercial availability. The corneal sensitivity thresholds are determined by recording the longest length of nylon filament that evokes a mechanical touch sensation on the corneal surface. Thresholds in mm units can be converted into pressure units $\left(\mathrm{g} / \mathrm{mm}^{2}\right)$ by referring to the calibration table provided by the manufacturer (Luneau Technology, Prunay-le-Gilon, France). However, the range of pressure values displayed in the calibration table for the $0.12 \mathrm{~mm}$ diameter filament $\left(0.4-10.3 \mathrm{~g} / \mathrm{mm}^{2}\right)$ differs from those reported in studies that conducted validation tests on the same device (Millodot and Larsen: $1-13.4 \mathrm{~g} / \mathrm{mm}^{2}$, Lowther and Hill: $4-354 \mathrm{mg} / \mathrm{mm}^{2}$, Norn: $0.9-7.1 \mathrm{~g} / \mathrm{mm}^{2}$, Lawrenson and Ruskell: $2.2-75.2 \mathrm{~g} /$ $\mathrm{mm}^{2}$, Golebiowski et al.: $0.5-23.1 \mathrm{~g} / \mathrm{mm}^{2}$, Chao et al.: $0.6-56.2 \mathrm{~g} / \mathrm{mm}^{2}$ ) [7, 19-22]. A possible explanation for the lack of agreement between the manufacturer and published studies is the difference in the techniques used to determine exerted pressure, and the differing levels of ambient room humidity where measurements were conducted. Several authors have suggested relative humidity levels may influence the rigidity of the nylon filament $[6,7,21]$, thereby altering the exerted pressure and leading to variations from those stated in the manufacturer's table. If correct, humidity-induced fluctuations in exerted pressure will have implications on the accuracy and precision of corneal sensitivity measurements using the COBO. The aim of this study was to examine the influence of relative humidity (\% $\mathrm{RH})$ levels on the pressure exerted by the COBO instrument.

\section{Methods}

A new, $0.12 \mathrm{~mm}$ nominal diameter, nylon filament was fitted into a COBO instrument (Model L12 No 8796, Luneau Technology, Prunay-le-Gilon, France) according to the manufacturer's guidelines. The instrument was placed in an environment-controlled chamber (Electro-tech systems Inc., PA, USA), where the \%RH level could be adjusted between $20 \%$ and $80 \%$. The chamber temperature was kept constant at $25^{\circ} \mathrm{C}$.

After $12 \mathrm{~h}$ in the chamber to allow for acclimatisation for the thread, the instrument was removed and positioned vertically above, and perpendicular to, the base plate of an analytical balance (Mettler-Toledo AB265; precision $\pm 0.0001 \mathrm{~g}$ ). The instrument was held in position, using a

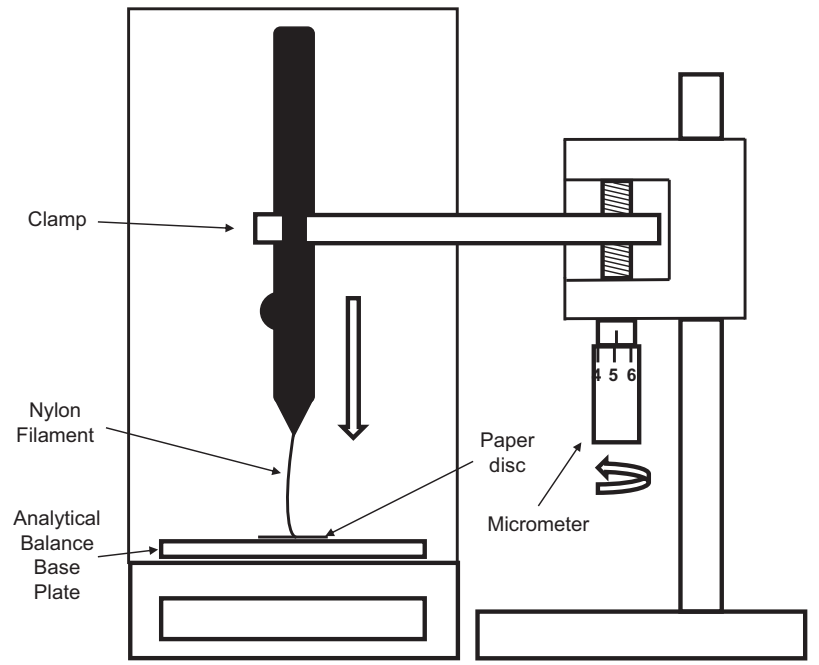

Fig. 1 Apparatus setup for the exerted pressure measurements

combination of clamps, multi-axis stage (World Precision Instruments, FL, USA) and cam seam micrometre (Mitutoyo, IL, USA: precision $\pm 0.01 \mathrm{~mm}$ ), to provide accurate centring and lowering of the instrument towards the base plate (Fig. 1). With the nylon filament extended to a specific length and using the micrometre, the instrument was gradually lowered towards the plate until contact was made by the filament tip. Initial contact between the filament and base plate was confirmed by observing a $0.0001-3 \mathrm{~g}$ increase in balance reading. Starting at the $60 \mathrm{~mm}$ filament length, measurements of applied filament force (in grams) were recorded over a total lowering distance of $1 \mathrm{~mm}$, in $0.1 \mathrm{~mm}$ step increments through fine manual adjustments of the micrometre. Measurements were made $30 \mathrm{~s}$ after each adjustment of distance to allow for the settling of the filament on the balance. Filament length was then reduced by $5 \mathrm{~mm}$ and measurement procedure repeated, down to the 10 $\mathrm{mm}$ filament length. A small disc of paper was placed on the balance plate to prevent filament slippage during measurements. The $\mathrm{COBO}$ was returned to the environment chamber to re-acclimatise, and the procedure repeated for a total of five repeated measurements at that $\% \mathrm{RH}$ level. Once all five measurements had been completed, the \%RH level was adjusted, and the sequence repeated. To include all \% RH levels between $20 \%$ and $80 \%$, the $\%$ RH setting was changed in $10 \%$ steps, and in a randomised order.

The diameter of the nylon filament for each \% RH setting was also measured by placing the instrument with filament fully extended and flat on the stage of a profile projector (Mitutoyo Model PJ300, Japan, precision $\pm 0.001 \mathrm{~mm}$ ). With a magnified view of the filament tip centred on the projector screen, ten successive thickness measurements $(d)$ were made by manual movement of $X-Y$ stage. Force measurements were then divided by the average measured cross-sectional area $\left(\pi_{\mathrm{x}}[d / 2]^{2}\right)$ of the nylon filament in $\mathrm{mm}^{2}$, 
Fig. 2 Changes in applied force (log scale) produced with lowering distance, for $10 \mathrm{~mm}$ and $60 \mathrm{~mm}$ filament lengths $(0.12 \mathrm{~mm}$ nominal filament diameter) at $20 \% \mathrm{RH}$ and $80 \%$ RH levels. Error bars represent one standard deviation. *denotes significant difference $(p<0.05)$ from subsequent data points

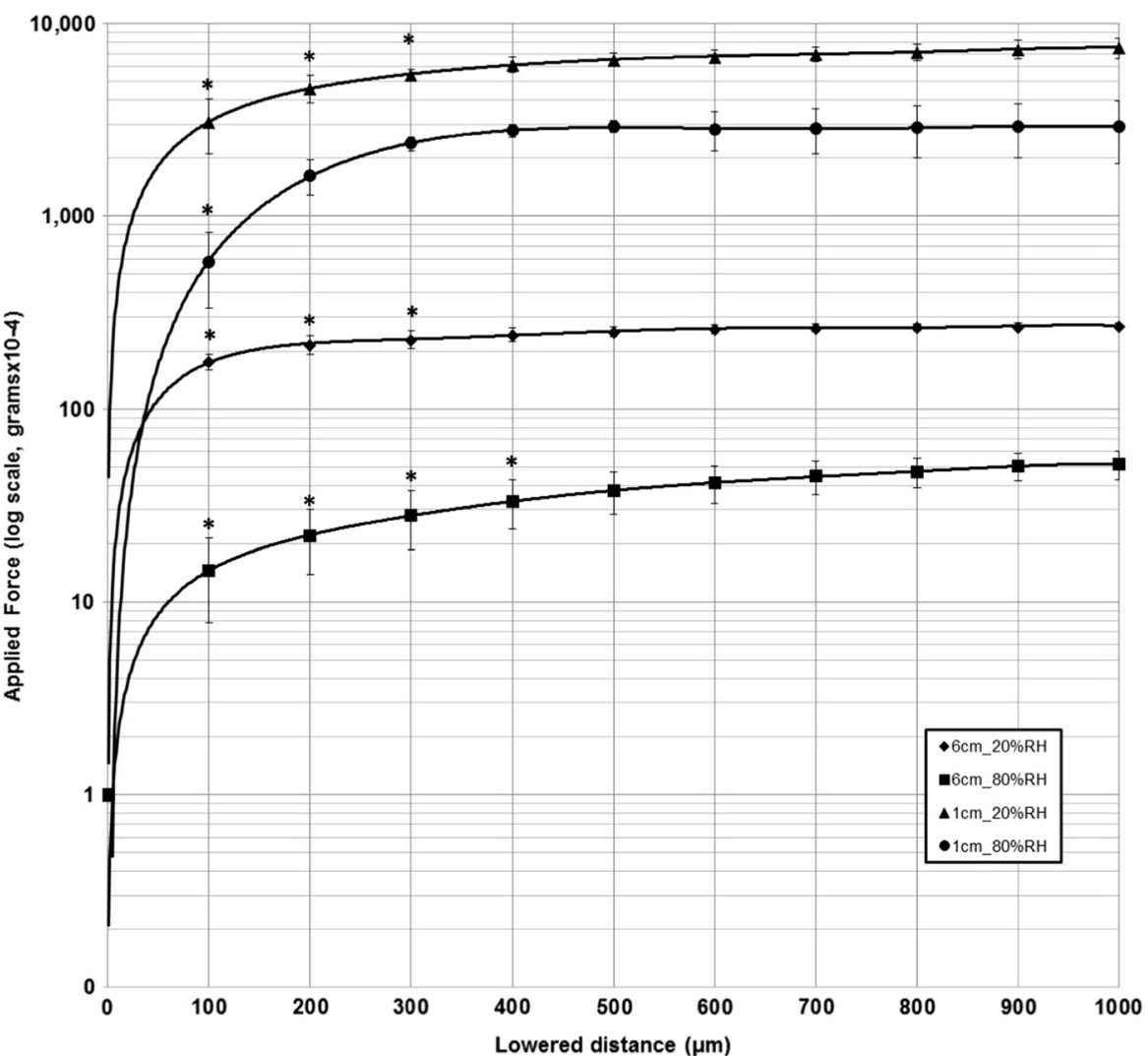

which gave the pressure measurement for the filament length $\left(\mathrm{g} / \mathrm{mm}^{2}\right)$.

Measurements of applied filament force were also conducted for a previously used $0.08 \mathrm{~mm}$ nominal diameter nylon filament fitted within the $\mathrm{COBO}$ instrument using the same testing procedure. However, measurements were made only for 10-60 mm filament lengths, in $10 \mathrm{~mm}$ steps, and for $\%$ RH settings between $20 \%$ and $80 \%$, in $20 \%$ steps.

\section{Statistical analysis}

To compare the changes in applied force across lowered distance for the $0.08 \mathrm{~mm}$ and $0.12 \mathrm{~mm}$ instruments, oneway ANOVA and post-hoc Tukey's range tests were carried out on data from each nylon length and \%RH (SPSSv25, IBM Corp., NY, USA). All force measurements that were found to be not significantly different, over a lowering distance range for a particular thread length and \% RH level, were averaged and taken as the mean applied force for that filament length. Changes in mean applied force across the tested range of \%RH levels for each filament length were then compared using a separate one-way ANOVA with post-hoc Tukey's range test. Filament thicknesses at each \% $\mathrm{RH}$ level were compared using one-way ANOVA with post-hoc Bonferroni correction, for $0.08 \mathrm{~mm}$ and $0.12 \mathrm{~mm}$ nominal diameter filaments. A $p$-value $<0.05$ denoted statistical significance.

\section{Results}

Applied force increased initially with changes in lowering distance of the instrument onto the microbalance, followed by a plateau of force measurements, for all filament lengths. Fig. 2 illustrates the changes in applied force for the 10 $\mathrm{mm}$ and $60 \mathrm{~mm}$ filament lengths $(0.12 \mathrm{~mm}$ nominal diameter filament), at the upper (80\%) and lower (20\%) \%RH levels. The start position for the plateau of force measurements varied for different filament lengths and \% RH levels, and ranged between $200 \mu \mathrm{m}$ and $800 \mu \mathrm{m}$ lowering distance.

There were significant changes in applied force with alterations in chamber \%RH levels, for all filament lengths $(p<.001)$ (Fig. 3). Applied force decreased logarithmically with stepwise increases in \%RH level in both filament diameters tested. Reductions in force appeared greater in the thicker $(0.12 \mathrm{~mm})$, compared to thinner $(0.08 \mathrm{~mm})$, nominal filament diameters over the measured \% RH range.

Mean thickness measured for the $0.08 \mathrm{~mm}$ and $0.12 \mathrm{~mm}$ nominal filament diameters were $0.086 \pm 4 \mathrm{~mm}$ and $0.127 \pm$ $1 \mathrm{~mm}$, respectively. There was no significant change in 
Fig. 3 Changes in applied force (log scale) produced with $\% \mathrm{RH}$ levels (20-80\%) for $10-60 \mathrm{~mm}$ filament lengths $(0.12 \mathrm{~mm}$ and $0.08 \mathrm{~mm}$ nominal filament diameters). Note: impact of altering humidity levels on applied force appears more significant for the thicker $0.12 \mathrm{~mm}$ (solid lines) than thinner $0.08 \mathrm{~mm}$ (dashed lines) filament. Error bars represent one standard deviation

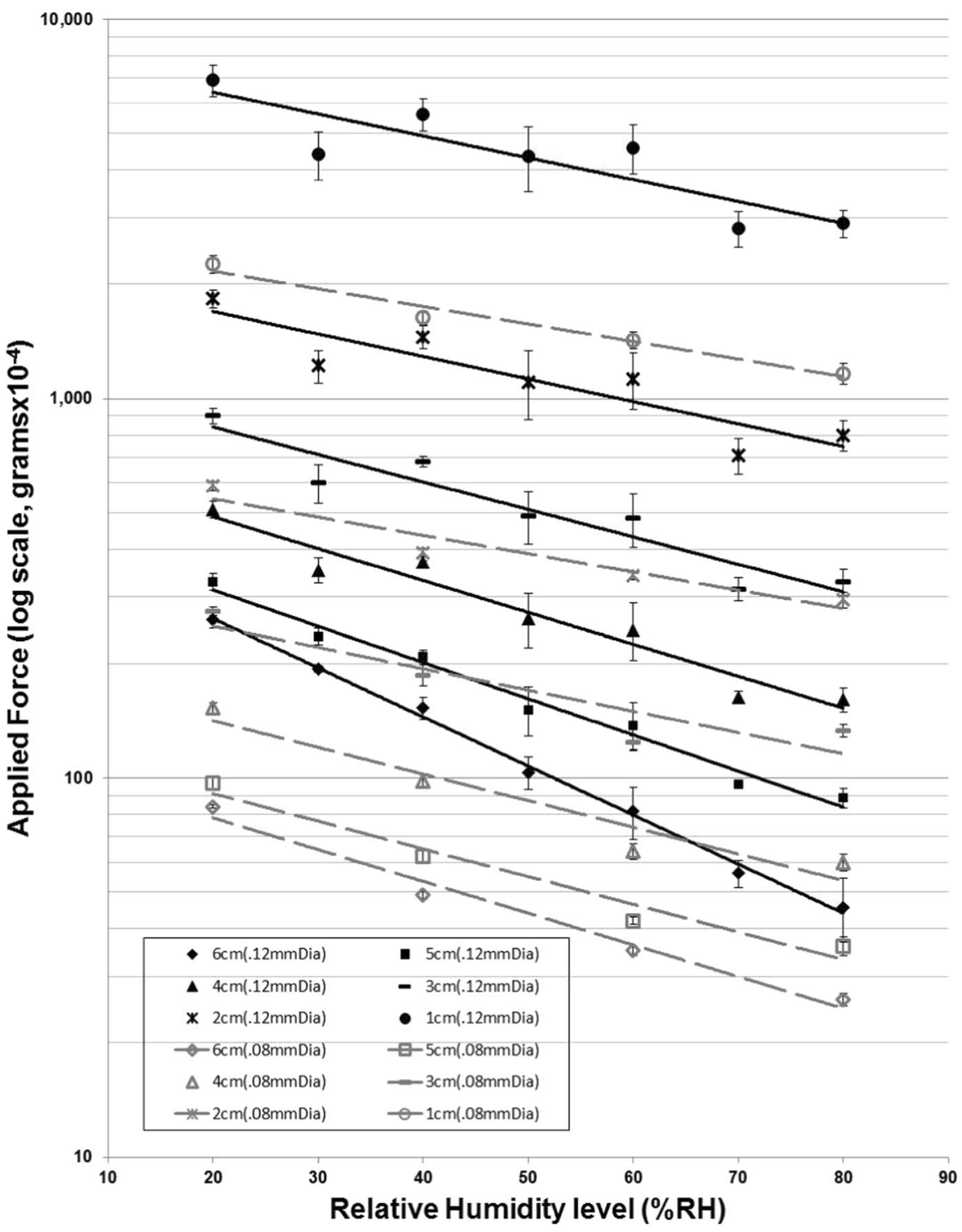

filament thickness over \% RH range for both filament diameters $(p>0.05)$.

Table 1 displays calculated exerted pressure $\left(\mathrm{g} / \mathrm{mm}^{2}\right)$ in homogenous subsets $($ alpha $=0.05)$ for all measured filament lengths and \%RH levels. Fig. 4a-c illustrate increased bending of the $0.12 \mathrm{~mm}$ filament under its own weight at higher \% RH levels when the instrument was held in the horizontal position.

\section{Discussion}

For the $0.08 \mathrm{~mm}$ and $0.12 \mathrm{~mm}$ nominal diameter filaments, significant changes in exerted force were observed for the same filament length following exposure of the COBO instrument to different levels of humidity, confirming previous suggestions that the force exerted by the $\mathrm{COBO}$ varies with ambient room humidity levels. On average, force decreased by $12 \%$ and $15 \%$ with each $10 \%$ step increase in $\% \mathrm{RH}$ levels, for the $0.08 \mathrm{~mm}$ and $0.12 \mathrm{~mm}$ nominal filament diameters, respectively. In addition, gradients of force versus \% RH slopes for each filament length appear steeper for the $0.12 \mathrm{~mm}$ compared to the $0.08 \mathrm{~mm}$ filament, particularly at longer filament lengths (Fig. 3), which suggests alterations in ambient humidity levels have a greater impact on the thicker diameter filament.

A reduction in exerted force with exposure to elevated humidity levels indicates a gradual loss of material rigidity within the nylon filament. This is clearly seen by the increased bending of the filament under its own weight when the instrument is held in the horizontal position (Fig. $4 \mathrm{a}-\mathrm{c}$ ). We suspect this reduction in filament rigidity is due to the absorption of moisture by the nylon material. However, no significant changes in filament diameter were 
Table 1 Conversion table for $0.12 \mathrm{~mm}$ nominal diameter filament

\begin{tabular}{|c|c|c|c|c|c|c|c|}
\hline \multirow[t]{2}{*}{ Filament length $(\mathrm{cm})$} & \multirow[t]{2}{*}{ Humidity $(\% \mathrm{RH})$} & \multicolumn{6}{|c|}{ Exerted pressure $\left(\mathrm{g} / \mathrm{mm}^{2}\right)$} \\
\hline & & 1 & 2 & 3 & 4 & 5 & 6 \\
\hline \multirow[t]{7}{*}{6} & 80 & $0.36(0.07)$ & & & & & \\
\hline & 70 & $0.44(0.04)$ & & & & & \\
\hline & 60 & & $0.64(0.10)$ & & & & \\
\hline & 50 & & & $0.81(0.08)$ & & & \\
\hline & 40 & & & & $1.21(0.08)$ & & \\
\hline & 30 & & & & & $1.53(0.03)$ & \\
\hline & 20 & & & & & & $2.06(0.10)$ \\
\hline \multirow[t]{7}{*}{5.5} & 80 & $0.51(0.06)$ & & & & & \\
\hline & 70 & $0.61(0.03)$ & & & & & \\
\hline & 60 & & $0.88(0.07)$ & & & & \\
\hline & 50 & & $0.96(0.13)$ & & & & \\
\hline & 40 & & & $1.42(0.07)$ & & & \\
\hline & 30 & & & & $1.61(0.09)$ & & \\
\hline & 20 & & & & & $2.26(0.09)$ & \\
\hline \multirow[t]{7}{*}{5} & 80 & $0.70(0.04)$ & & & & & \\
\hline & 70 & $0.76(0.01)$ & & & & & \\
\hline & 60 & & $1.09(0.16)$ & & & & \\
\hline & 50 & & $1.20(0.18)$ & & & & \\
\hline & 40 & & & $1.65(0.06)$ & & & \\
\hline & 30 & & & $1.86(0.10)$ & & & \\
\hline & 20 & & & & $2.59(0.14)$ & & \\
\hline \multirow[t]{7}{*}{4.5} & 80 & $0.91(0.07)$ & & & & & \\
\hline & 70 & $0.94(0.04)$ & & & & & \\
\hline & 60 & & $1.40(0.20)$ & & & & \\
\hline & 50 & & $1.56(0.21)$ & & & & \\
\hline & 40 & & & $2.20(0.07)$ & & & \\
\hline & 30 & & & $2.16(0.21)$ & & & \\
\hline & 20 & & & & $3.07(0.24)$ & & \\
\hline \multirow[t]{7}{*}{4} & 80 & $1.27(0.09)$ & & & & & \\
\hline & 70 & $1.29(0.05)$ & & & & & \\
\hline & 60 & & $1.94(0.33)$ & & & & \\
\hline & 50 & & $2.07(0.34)$ & & & & \\
\hline & 40 & & & $2.91(0.03)$ & & & \\
\hline & 30 & & & $2.78(0.22)$ & & & \\
\hline & 20 & & & & $4.03(0.20)$ & & \\
\hline \multirow[t]{7}{*}{3.5} & 80 & $1.86(0.08)$ & & & & & \\
\hline & 70 & $1.82(0.14)$ & & & & & \\
\hline & 60 & & $2.68(0.52)$ & & & & \\
\hline & 50 & & $2.85(0.42)$ & & & & \\
\hline & 40 & & & $4.05(0.13)$ & & & \\
\hline & 30 & & & $3.61(0.31)$ & & & \\
\hline & 20 & & & & $5.27(0.30)$ & & \\
\hline \multirow[t]{4}{*}{3} & 80 & $2.58(0.20)$ & & & & & \\
\hline & 70 & $2.48(0.17)$ & & & & & \\
\hline & 60 & & $3.81(0.61)$ & & & & \\
\hline & 50 & & $3.86(0.61)$ & $3.86(0.61)$ & & & \\
\hline
\end{tabular}


Table 1 (continued)

\begin{tabular}{|c|c|c|c|c|c|c|c|}
\hline \multirow[t]{2}{*}{ Filament length $(\mathrm{cm})$} & \multirow[t]{2}{*}{ Humidity $(\% \mathrm{RH})$} & \multicolumn{6}{|c|}{ Exerted pressure $\left(\mathrm{g} / \mathrm{mm}^{2}\right)$} \\
\hline & & 1 & 2 & 3 & 4 & 5 & 6 \\
\hline & 30 & & & $4.73(0.55)$ & $4.73(0.55)$ & & \\
\hline & 40 & & & & $5.38(0.17)$ & & \\
\hline & 20 & & & & & $7.09(0.35)$ & \\
\hline \multirow[t]{7}{*}{2.5} & 80 & $3.78(0.39)$ & & & & & \\
\hline & 70 & $3.60(0.32)$ & & & & & \\
\hline & 60 & & $5.40(0.83)$ & & & & \\
\hline & 50 & & $5.80(0.89)$ & & & & \\
\hline & 30 & & $6.48(0.54)$ & $6.48(0.54)$ & & & \\
\hline & 40 & & & $7.45(0.26)$ & & & \\
\hline & 20 & & & & $9.79(0.54)$ & & \\
\hline \multirow[t]{7}{*}{2} & 80 & $6.30(0.59)$ & & & & & \\
\hline & 70 & $5.59(0.60)$ & & & & & \\
\hline & 60 & & $8.90(1.53)$ & & & & \\
\hline & 50 & & $8.71(1.79)$ & & & & \\
\hline & 30 & & $9.62(0.96)$ & $9.62(0.96)$ & & & \\
\hline & 40 & & & $11.48(0.79)$ & & & \\
\hline & 20 & & & & $14.44(0.76)$ & & \\
\hline \multirow[t]{7}{*}{1.5} & 80 & $11.72(1.18)$ & & & & & \\
\hline & 70 & $9.91(1.32)$ & & & & & \\
\hline & 60 & & $16.07(2.29)$ & & & & \\
\hline & 50 & & $15.90(2.96)$ & & & & \\
\hline & 30 & & $16.44(2.10)$ & $16.44(2.10)$ & & & \\
\hline & 40 & & & $20.27(1.69)$ & & & \\
\hline & 20 & & & & $24.99(0.93)$ & & \\
\hline \multirow[t]{7}{*}{1} & 80 & $22.83(1.85)$ & & & & & \\
\hline & 70 & $22.16(2.44)$ & & & & & \\
\hline & 60 & & $36.17(5.42)$ & & & & \\
\hline & 50 & & $34.33(6.60)$ & & & & \\
\hline & 30 & & $34.73(4.97)$ & & & & \\
\hline & 40 & & $44.40(4.33)$ & $44.40(4.33)$ & & & \\
\hline & 20 & & & $54.56(5.29)$ & & & \\
\hline
\end{tabular}

Mean $(\mathrm{SD})$ calculated exerted pressure $\left(\mathrm{g} / \mathrm{mm}^{2}\right)$ grouped in homogeneous subsets (alpha $\left.=0.05\right)$ over tested range of humidity levels $(\% \mathrm{RH})$, for each filament length

detected across the range of humidity levels tested. The absence of a measurable thickness change indicates that the filament's cross-sectional area remains relatively constant over a wide range of humidity levels, and that fluctuations in ambient room humidity has a minimal impact on the stimulus footprint on the corneal surface during corneal sensitivity assessment.

In this study, we observed a gradual increase in exerted force as the $\mathrm{COBO}$ was advanced towards the microbalance scale, following contact with the plate. This was not surprising, given our measurement technique and the flexural properties of the nylon filament. The guideline for measuring corneal sensitivity threshold provided by Cochet and
Bonnet [23] is to advance the filament onto the corneal surface until a $4 \%$ flexure or $5^{\circ}$ bend is observed. Although this criterion provides a repeatable method for determining exerted force, it is not practical, as there is no means by which an operator can accurately measure filament bend angle. An alternate criterion reported in studies is to advance the instrument until a slight bend in the filament is observed. However, this endpoint is subjective and is likely to result in poor stimulus repeatability for the same filament length, given the initial pattern of exerted force change observed in this study (Fig. 2). Changes in exerted force, however, were found to plateau onwards from a specific lowering distance for each filament length and \% RH level. 


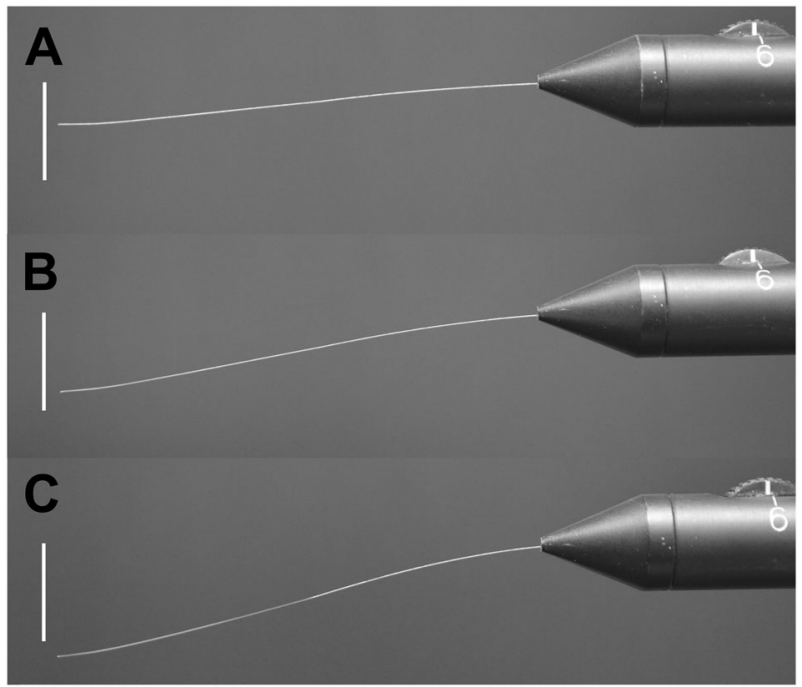

Fig. 4 Appearance of $0.12 \mathrm{~mm}$ nominal diameter filament at (a) $20 \%$, (b) $50 \%$, and (c) $80 \% \% \mathrm{RH}$ levels. Bar (vertical) $=10 \mathrm{~mm}$

Therefore, we recommend the instrument be advanced onto the corneal surface by at least $1 \mathrm{~mm}$ to provide consistency in the stimulus intensity during threshold measurement. We observed that a $1 \mathrm{~mm}$ lowering distance corresponded to a significant bend in the nylon filament.

The exerted force and calculated pressure values presented in Table 1 were significantly greater than those provided in the manufacturer's calibration table for the $0.12 \mathrm{~mm}$ diameter instrument. This disparity in pressure values is likely due to the effects of humidity on the nylon filament. It may also be due to differences in the method used to measure and calculate exerted force. That is, our table represents the peak values for each nylon length (i.e. plateau of force) at each measured humidity level, whereas the manufacturer's table presumably describes pressure values at a $5^{\circ}$ bend in the filament at $55 \%$ humidity level.

Interestingly, although not presented here in this report, we observed a gradual lowering of measured force with repeated measurement, using our in vitro technique for most length/humidity combinations. However, no consistent pattern could be determined and hence the cause of this decreasing drift in pressure for the same filament and \% RH on repeat measurements is unknown. However, previous authors have suggested the strength of the nylon filament may decrease with instrument use over time. We cannot estimate the period of normal use that our testing procedure represents. Nevertheless, replacement of the nylon filament after long periods of use is recommended to ensure consistency in the exerted pressure and to avoid drifts in sensitivity thresholds. Alternatively, Chao and colleagues [19] suggests the recalibration of an instrument's conversion table before use to enable the accurate ocular surface sensitivity measurement.
A limitation of this study is that we did not examine whether the alterations in applied pressure from varying ambient humidity levels were clinically significant. However in the study by Chao et al.[19], they report a correlation of repeatability $(\mathrm{CoR})$ of $\pm 0.06 \mathrm{~g} / \mathrm{mm}^{2}$ for same-day corneal sensitivity thresholds, for the COBO instrument. Taking this CoR value as the 'just noticeable difference' for corneal sensitivity, a change in \% RH that altered the exerted pressure by greater than $0.06 \mathrm{~g} / \mathrm{mm}^{2}$ for the same filament length would then result in a clinically detectable difference. In the $0.12 \mathrm{~mm}$ instrument, this magnitude of pressure change is seen for all but a few $10 \%$ stepwise humidity change and filament lengths (Table 1). Therefore, a 10\%RH change ambient room humidity is likely to result in a clinically detectable and hence significant difference in corneal threshold. An additional limitation is that we did not examine whether altering humidity levels have an impact on exerted pressure when the COBO is stored in its case. It is, however, recommended that the instrument is kept within the case when not in use. Furthermore, liquids, such as glutaraldehyde or other solutions compatible with nylon, are recommended by the manufacturer to disinfect the filament tip following use. Contact between such liquids and the filament tip would presumably impact the filament rigidity; however, this needs to be confirmed. Furthermore, we did not examine the influence of ambient room temperature on the exerted pressure, and this requires further investigation.

In summary, this study confirms previous suggestions that the rigidity of the COBO nylon filament is affected by ambient room humidity levels, particularly for the thicker $0.12 \mathrm{~mm}$ nylon filament. One implication of this is a potential reduction in the repeatability of corneal sensitivity measurements. We recommend the monitoring of ambient room humidity levels while conducting the assessment of corneal sensitivity, and of maintaining it at a constant level to avoid any confounding variations in exerted filament pressure. If the control of humidity level is not possible, we provide a table for converting filament lengths to exerted pressure (Table 1) that includes changes in ambient room humidity, for the $0.12 \mathrm{~mm}$ filament diameter instrument.

\section{Summary}

\section{What was known before:}

- The COBO aesthesiometer is the current standard in corneal sensitivity assessment.

- Several authors have suggested relative humidity levels may influence the rigidity of the nylon filament, thereby altering the exerted pressure and accuracy of the instrument. 


\section{What this study adds:}

- Our study confirms previous suggestions that the rigidity of the COBO nylon filament is affected by ambient room humidity levels, which have implications of sensitivity measurements.

- We provide a correction table for converting filament lengths to pressure for a range of relative humidity levels.

Acknowledgements The authors wish to thank the Choh Lab for the use of equipment, and Ms Contanze Bayha from the Applied Science University, Aalen, Germany, for her assistance in measuring the filament force. This study has been previously presented at the Association for Research in Vision and Ophthalmology (ARVO), May 2017, Baltimore, MD, USA.

\section{Compliance with ethical standards}

Conflict of interest No conflicting relationship exists for any author. This research was supported under the Australia Awards-Endeavour Research Fellowship program.

\section{References}

1. Stern M, Beuerman R, Fox R, Gao J, Mitcheff A, Pflugfelder S. The pathology of dry eye: the interaction between the ocular surface and lacrimal Glands. Cornea. 1998;17:584.

2. Willshire C, Buckley RJ, Bron AJ. Central connections of the lacrimal functional unit. Cornea. 2017;36:898-907.

3. Beuerman RW, Schimmelpfennig B. Sensory denervation of the rabbit cornea affects epithelial properties. Exp Neurol. 1980;69:196-201.

4. Ueno H, Ferrari G, Hattori T, et al. Dependence of corneal stem/ progenitor cells on ocular surface innervation. Invest Ophthal Vis Sci. 2012;53:867-72.

5. Belmonte C, Acosta MC, Schmelz M, Gallar J. Measurement of corneal sensitivity to mechanical and chemical stimulation with a $\mathrm{CO}_{2}$ esthesiometer. Invest Ophthal Vis Sci. 1999;40:513-9.

6. Murphy PJ, Patel S, Marshall J. A new non-contact corneal aesthesiometer (NCCA). Ophthalmic Physiol Opt. 1996;16:101-7.

7. Golebiowski B, Papas E, Stapleton F. Assessing the sensory function of the ocular surface: Implications of use of a non-contact air jet aesthesiometer versus the Cochet-Bonnet aesthesiometer. Exp Eye Res. 2011;92:408-13.
8. Millodot M, O’Leary DJ. Corneal fragility and its relationship to sensitivity. Acta Ophthalmol. 1981;59:820-6.

9. Murphy PJ, Lawrenson JG, Patel S, Marshall J. Reliability of the non-contact corneal aesthesiometer and its comparison with the Cochet-Bonnet aesthesiometer. Ophthalmic Physiol Opt. 1998;18:532-9.

10. Cruzat A, Hamrah P, Cavalcanti B, Zheng L, Colby K, PavanLangston D. Corneal reinnervation and sensation recovery in patients with Herpes Zoster Ophthalmicus: an in vivo and ex vivo study of corneal nerves. Cornea. 2016;35:619-25.

11. Mandathara P, Stapleton F, Kokkinakis J, Willcox M. Pilot study of corneal sensitivity and its association in keratoconus. Cornea. 2017;36:163-8.

12. Ornek N, Dag E, Ornek K. Corneal sensitvity and tear function in neurodegenerative diseases. Curr Eye Res. 2015;40:423-8.

13. Bouheraoua N, Hrarat L, Parsa CF, et al. Decreased corneal sensation and subbasal nerve density, and thinned corneal epithelium as a result of 360-degree laser retinopexy. Ophthalmology. 2015;122:2095-102.

14. Li Q, Fu T, Yang J, Wang Q, Li Z. Ocular surface changes after strabismus surgery with different incisions. Graefes Arch Clin Exp Ophthalmol. 2015;253:431-8.

15. Sauvageot P, Julio G, Alvarez de Toledo J, Charoenrook V, Barraquer R. Femtosecond laser-assisted laser in situ keratomileusis versus photorefractive keratectomy: Effect on ocular surface condition. J Cataract Refract Surg. 2017;43:167-73.

16. Julio G, Campos P, Pujol P, Munguia A, Mas-Aixala E. Determining factors for fast corneal sensitivity recovery after pterygium excision. Cornea. 2016;35:1594-9.

17. Golebiowski B, Chao C, Stapleton F, Jalbert I. Corneal nerve morphology, sensitivity and tear neuropeptides in contact lens wear. Optom Vis Sci. 2017;94:534-42.

18. Hiraoka T, Kaji Y, Okamoto F, Oshika T. Corneal sensation after overnight orthokeratology. Cornea. 2009;28:891-5.

19. Chao C, Stapleton F, Badarudin E, Golebiowski B. Ocular surface sensitivity repeatability with Cochet-Bonnet esthesiometer. Optom Vis Sci. 2015;92:183-9.

20. Lowther GE, Hill RM. Sensitivity threshold of the lower lid margin in the course of adaptation to contact lenses. Am J Optom Arch Am Acad Optom. 1968;45:587-94.

21. Millodot M, Larson W. Effect of bending of the nylon thread of the Cochet-Bonnet aesthesiometer upon the recording pressure. Contact Lens J. 1963;1:5-7. 28

22. Norn MS. Conjunctival sensitivity in normal eyes. Acta Ophthalmol. 1973;51:58-66.

23. Cochet P, Bonnet R. Corneal esthesiometry: clinical measurement and physiological and pathological changes. La Clin Ophtalomogique. 1960;4:3-17. (French). 